\title{
MiR-585-3p suppresses tumor proliferation and migration by directly targeting CAPN9 in high grade serous ovarian cancer
}

\author{
Xiaoyuan Lu ${ }^{1 \dagger}$, Guilin $\mathrm{Li}^{2+}$, Sicong Liu ${ }^{3}$, Haihong Wang ${ }^{1}$ and Buze Chen ${ }^{1,4^{*}}$ (D)
}

\begin{abstract}
Background: Aberrant expression of microRNAs (miRNAs) contributes to the development of high grade serous ovarian cancer (HGSOC). However, the molecular mechanism by which miRNA-585-3p mediates high-grade serous ovarian carcinogenesis is unclear. This study aims to investigate the specific mechanism of action of miR-585-3p in HGSOC.

Methods: Expression of miR-585-3p in HGSOC tissues and cell lines was detected by qRT-PCR. Cell viability and migration were detected using MTT and transwell system. The expression of target genes and target proteins of miR$585-3 p$ was detected by dual luciferase reporter assay and western blot.

Results: The expression of miR-585-3p was significantly lower in HGSOC tissues and cells than in normal ovarian tissues and cell lines. In HGSOC tissues, CAPN9 expression was inversely correlated with miR-585-3p expression. MiR585-3p inhibited the proliferation and migration of HGSOC cells. MiR-585-3p bound to the 3'-untranslated region (UTR) of CAPN9 and inhibits CAPN9 expression. Overexpression of CAPN9 reduced the inhibitory effect of miR-585-3p in $\mathrm{HGSOC}$ cells.
\end{abstract}

Conclusions: miR-585-3p is significantly down-regulated in HGSOC tissues and cell lines. MiR-585-3p inhibits the proliferation and migration of HGSOC cells by targeting CAPN9.

Keywords: Ovarian cancer, miR-585-3p, CAPN9, Bioinformatics, Cell biology

\section{Introduction}

In worldwide, there are only an estimated 239,000 new cases of ovarian cancer (OC) and 152,000 deaths each year [1]. OC has the highest mortality rate of all gynecologic tumors, which is one of the main causes of death from gynecologic malignancies in China [2, 3]. OC exhibits a wide range of clinical, histopathological, and molecular heterogeneity and has been put into several subtypes, about $70 \%$ of which are high grade serous

\footnotetext{
*Correspondence: zku4ba@163.com

${ }^{\dagger}$ Xiaoyuan Lu and Guilin Li contributed equally.

1 Department of Gynecology, The Affiliated Hospital of Xuzhou Medical University, Jiangsu 221000, China

Full list of author information is available at the end of the article
}

ovarian cancer (HGSOC) subtypes [4]. 80\% of patients with HGSOC are diagnosed at an advanced stage for reasons including non-specific clinical symptoms and lack of reliable early detection biomarkers [3]. Development of targeted therapies to identify new molecular targets is necessary [5]. Therefore, it is important to investigate the mechanisms of ovarian carcinogenesis to improve the outcomes of patients with HGSOC.

MiRNAs play an important role in a variety of biological processes including cell proliferation, apoptosis, differentiation, and migration of tumors [6]. MiRNAs negatively regulate gene expression by inhibiting the translation of messenger RNA (mRNA) or by degrading mRNA [7]. The role of miR-585-3p has been explored in some cancers. Downregulated miR-585-3p promotes 
cell growth and proliferation in colon cancer by unregulated PSME3 [8]. MicroRNA-585 inhibits human glioma cell proliferation by directly targeting MDM2 [9]. MicroRNA-585, directly targeting MAPK1, suppresses gastric tumor proliferation and migration in gastric cancer [10]. These suggested that miR-585-3p may play an important role in HGSOC.

In this study, the expression levels and biological functions of miRNA-585-3p and its target genes in HGSOC were explored by bioinformatics and molecular biology methods. This study provides a theoretical basis for the treatment of HGSOC.

\section{Materials and methods}

\section{Samples and Cell lines}

Samples of tumor tissues and normal ovarian tissues from 10 HGSOC patients were collected at the Department of Obstetrics and Gynaecology of the Affiliated Hospital of Xuzhou Medical University. This study was approved by the ethics committee of Xuzhou Medical University Affiliated Hospital. All patients signed a written informed consent form. As shown in Table S1, the clinical characteristics of ten HGSOC patients were listed.

The OC cell lines SKOV3 and A2780, and the human ovarian surface epithelial cell line IOSE29, preserved in our laboratory, were used in this study. The cells were cultured with reference to the literature [5].

\section{QRT-PCR}

The miR-585-3p levels in 10 HGSOC tissue samples and the SKOV3, A2780 and IOSE29 cell lines were identified by qRT-PCR. The detailed steps were carried out according to the literature [5]. The primer sequences used were shown in Table S2.

\section{Plasmid construction and transfection}

MiR-585-3p mimics, miR-585-3p antisenses oligonucleotides (ASO) and control gene were designed and purchased from Sangon Biotech Company (https://www. sangon.com/; Shanghai, China). The sequences were listed in Table S2. Lipofectamine ${ }^{\circledR} 2000$ reagent (Thermo Fisher Scientific, Waltham, MA, USA) was used to transport miRNAs into the cells. The effect of CAPN9 overexpression on the proliferation of SKOV3 cell lines was studied using the pcDNA3.1 vector.

\section{Cell viability and migration}

Cell viability was checked by MTT assay [11]. Cell migration is detected by the Transwell system [12]. The detailed steps were carried out according to the literature [5].

\section{The targets of miR-585-3p}

TargetScan database (http://www.targetscan.org) and miRDB database (http://mirdb.org/) were used to predict putative targets of miR-585-3p [13-16].

\section{Analysis of miR-585-3p and CAPN9 interactions}

The detailed steps of dual luciferase reporter and western blot were carried out according to the literature [5]. CAPN9 3'-UTR sentinel mutagenesis using the SiteDirected Mutagenesis kit (Invitrogen, USA). For CAPN9 protein expression, an anti-CAPN9 antibody (Cat. No, PA5-54,197, Invitrogen, Carlsbad, CA, USA) was used at a dilution of 1:1000.

\section{Statistical analyses}

All calculations were performed using GraphPad Prism 7.0 software. Analyses between the two groups were compared using a two-tailed Student's t-test. Analyses between the three groups were conducted using one-way ANOVA with Student-Newman-Keuls test for post hoc comparisons. $\mathrm{P}<0.05$ indicated statistical significance.

\section{Results}

miR-585-3p was decreased in HGSOC tissues

As shown in Fig. 1, MiR-585-3p levels in tumor tissues were significantly lower than those in adjacent normal ovarian tissues $(1.00 \pm 0.000$ vs $0.36 \pm 0.042, \mathrm{P}<0.001)$.




High expression of miR-585-3p inhibited cell viability and migration

As shown in Fig. 2, SKOV3 and A2780 cells showed significantly lower levels of miR-585-3p compared to IOSE29 cells $(1.00 \pm 0.036$ vs $0.043 \pm 0.05, \quad \mathrm{P}<0.05$; $1.00 \pm 0.036$ vs $0.35 \pm 0.05, \mathrm{P}<0.05)$. As shown in Fig. $3 \mathrm{~A}$,

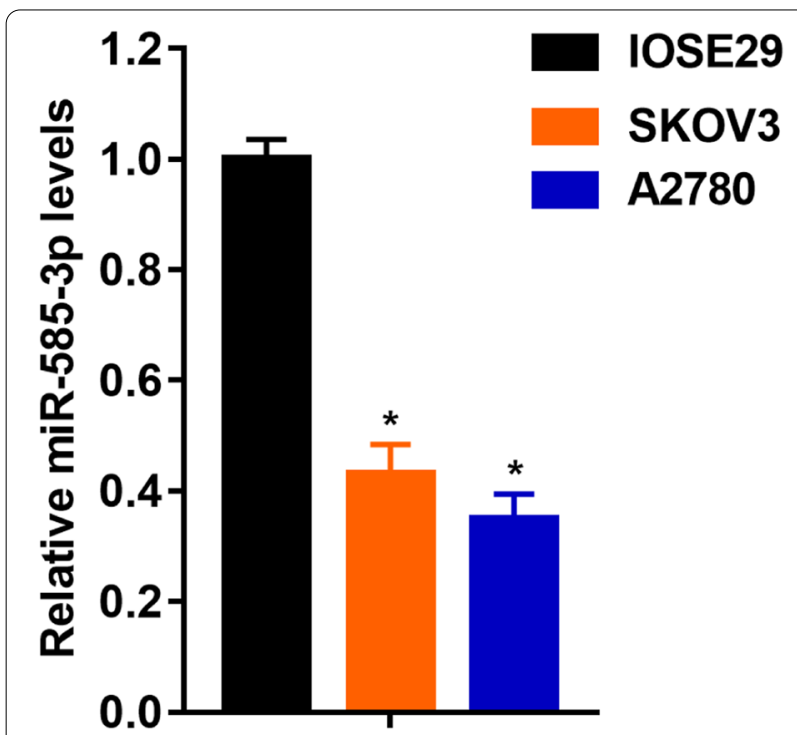

Fig. 2 Expression of miR-585-3p in human normal ovarian epithelial cells, ovarian cancer cell lines SKOV3 and A2780
miR-585-3p mimics increased levels of miR-585-3p in SKOV3 and A2780 cells $(1.00 \pm 0.10$ vs $5.80 \pm 0.08$, $\mathrm{P}<0.05 ; 1.00 \pm 0.08$ vs $5.70 \pm 0.09, \mathrm{P}<0.05)$. As shown in Fig. 3B, the viability of SKOV3 and A2780 cells was significantly inhibited within $72 \mathrm{~h}$ after transfection with miR-585-3p mimics $(390 \pm 0.5$ vs $190 \pm 0.6, \quad \mathrm{P}<0.05$; $510 \pm 0.4$ vs $230 \pm 0.4, \mathrm{P}<0.05)$. As shown in Fig. $3 \mathrm{C}$ and Fig. 3D, the number of migrating cells was significantly inhibited by miR-585-3p mimics.

\section{Low expression of miR-585-3p promoted cell viability and migration}

As shown in Fig. 4A, the miR-585-3p ASO inhibited levels of miR-585-3p in SKOV3 and A2780 cells (1.00 \pm 0.10 vs $0.70 \pm 0.09, \quad \mathrm{P}<0.05 ; \quad 1.00 \pm 0.11$ vs $0.59 \pm 0.10$, $\mathrm{P}<0.05)$. The viability of SKOV3 and A2780 cells was significantly promoted within $72 \mathrm{~h}$ after transfection with miR-585-3p ASO $(330 \pm 0.12$ vs $730 \pm 0.10, \mathrm{P}<0.05$; $390 \pm 0.13$ vs $830 \pm 0.12, \mathrm{P}<0.05$ ) (Fig. $4 \mathrm{~B}$ ). miR-585-3p ASO inhibited the number of migrating cells (Fig. $4 \mathrm{C}$ and Fig. 4D).

miR-585-3p directly targeted the CAPN9

As shown in Fig. 5, there were 24 common genes Common genes for miR-585-3p target genes predicted by TargetScan and miRDB. The common genes included C6orf136, HOGA1, MITF, CAPN9, CD34, RAVER2,
A

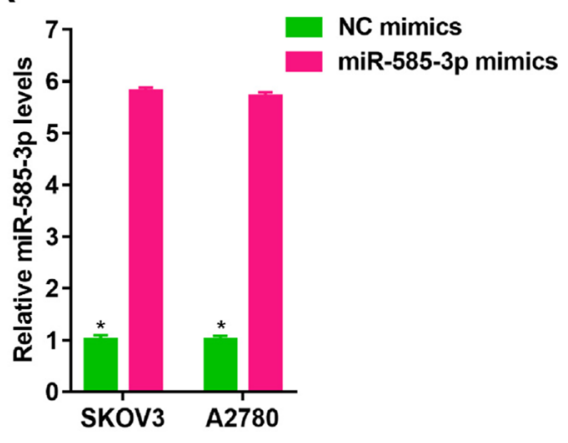

C



B

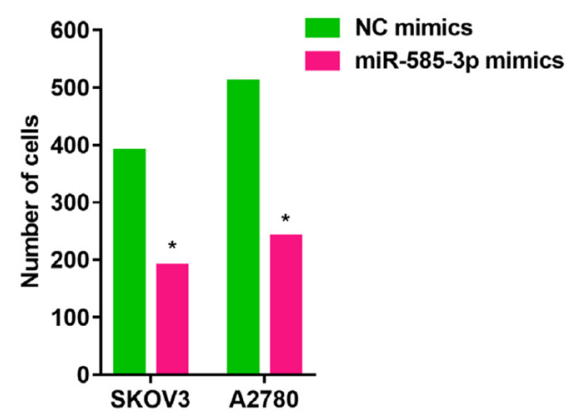

D

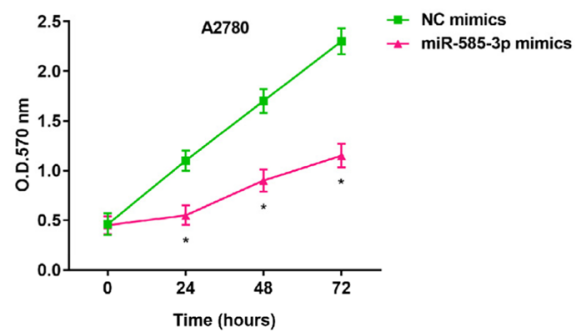

Fig. 3 Overexpression of miR-585-3p inhibited proliferation and migration of SKOV3 and A2780. A Expression of miR-585-3p in transfected cells. B Assessment of migration of transfected cells. C Cell growth of SKOV3. D Cell growth of A2780. ${ }^{* P}<0.05$ 

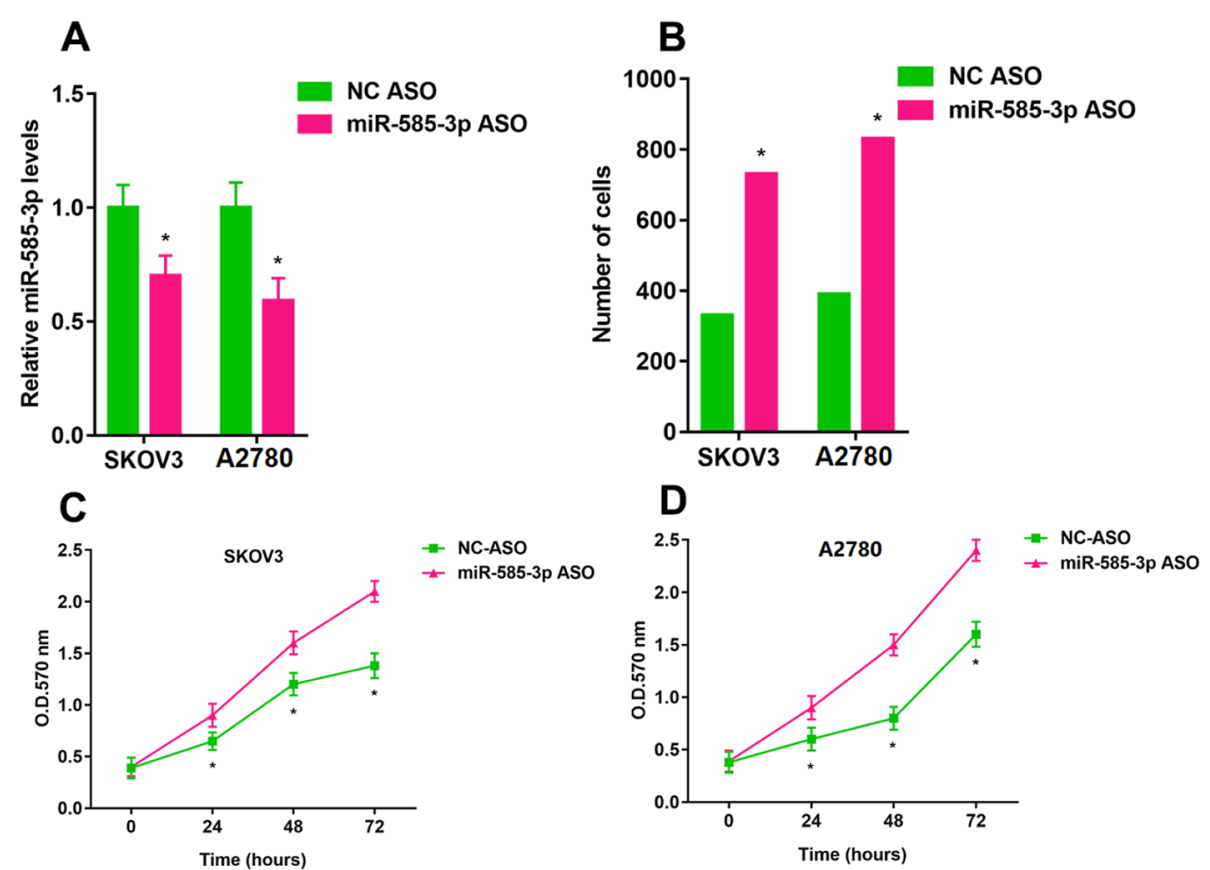

Fig. 4 Downregulation of miR-585-3p promotes proliferation and migration of SKOV3 and A2780. A Expression of miR-585-3p in transfected cells. B Assessment of migration of transfected cells. C Cell growth of SKOV3. D Cell growth of A2780. *P $<0.05$

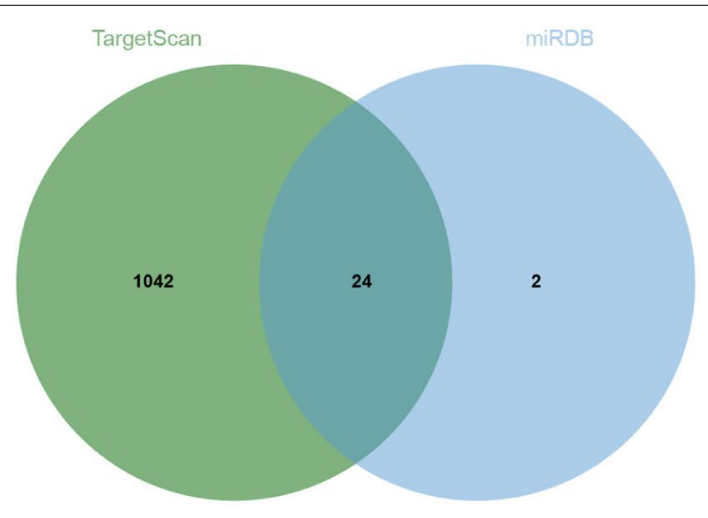

\begin{tabular}{|lll|}
\hline C6orf136 & GPR12 & FSCN1 \\
HOGA1 & SPOCK1 & TMEM102 \\
MITF & VMAC & HAVCR1 \\
CAPN9 & EXTL3 & DEDD \\
CD34 & ATP6V1C1 & TRPV3 \\
RAVER2 & ZFYVE26 & SMG1 \\
VPS4A & PRPF4 & SPCS3 \\
CDC25B & GPRIN1 & RNF213 \\
\hline
\end{tabular}

Fig. 5 Common genes for miR-585-3p target genes predicted by TargetScan and miRDB

VPS4A, CDC25B, GPR12, SPOCK1, VMAC, EXTL3, ATP6V1C1, ZFYVE26, PRPF4, GPRIN1, FSCN1, TMEM102, HAVCR1, DEDD, TRPV3, SMG1, SPCS3, and RNF213. The tumor tissues showed higher CAPN9 levels (Fig. 6A). CAPN9 expression was inversely correlated with miR-585-3p expression in HGSOC (Fig. 6B). As shown in Fig. 7A, the relationship between miR585-3p and CAPN9 was investigated using sentinel mutations. In SKOV3 cells, miR-585-3p targeted CAPN9 (Fig. 7B). CAPN9 overexpression reduced the inhibitory effect of miR-585-3p on SKOV3 and A2780 cells
(Fig. 7C). As shown in Fig. 7D, miR-585-3p transfection inhibited CAPN9 protein expression in SKOV3 cells. The results of the Western blot assay show the effect of transfection of pcDNA3.1-CANP9 in the SKOV3 cell line (Fig. 7E).

\section{Discussion}

In this study, MiR-585-3p was significantly lower expressed in tumor tissues and cell lines than in normal tissues and cell lines. miR-585-3p overexpression inhibited cell growth and migration of $\mathrm{OC}$ cell lines. 

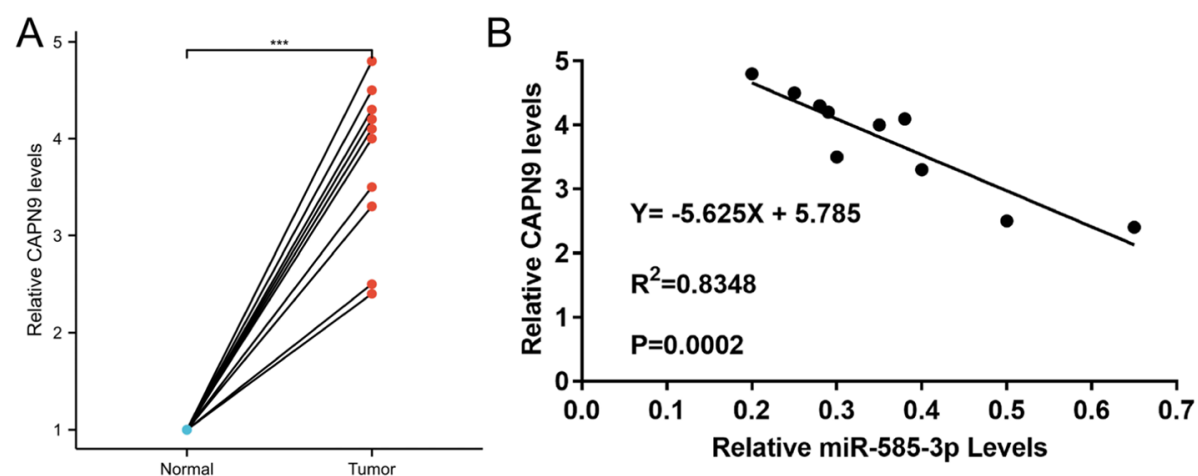

Fig. 6 The expression of CAPN9 in HGSOC tissues and correlation between CAPN9 and miR-585-3p. A CAPN9 levels in 10 HGSOC tissues and their matched adjacent normal tissues were assessed via qRT-PCR. Relative CAPN9 level tumor/normal (log2) is listed. B Pearson's correlation coefficient analysis revealed that miR-585-3p levels and CANP9 mRNA levels were inversely correlated in the HGSOC tissue samples

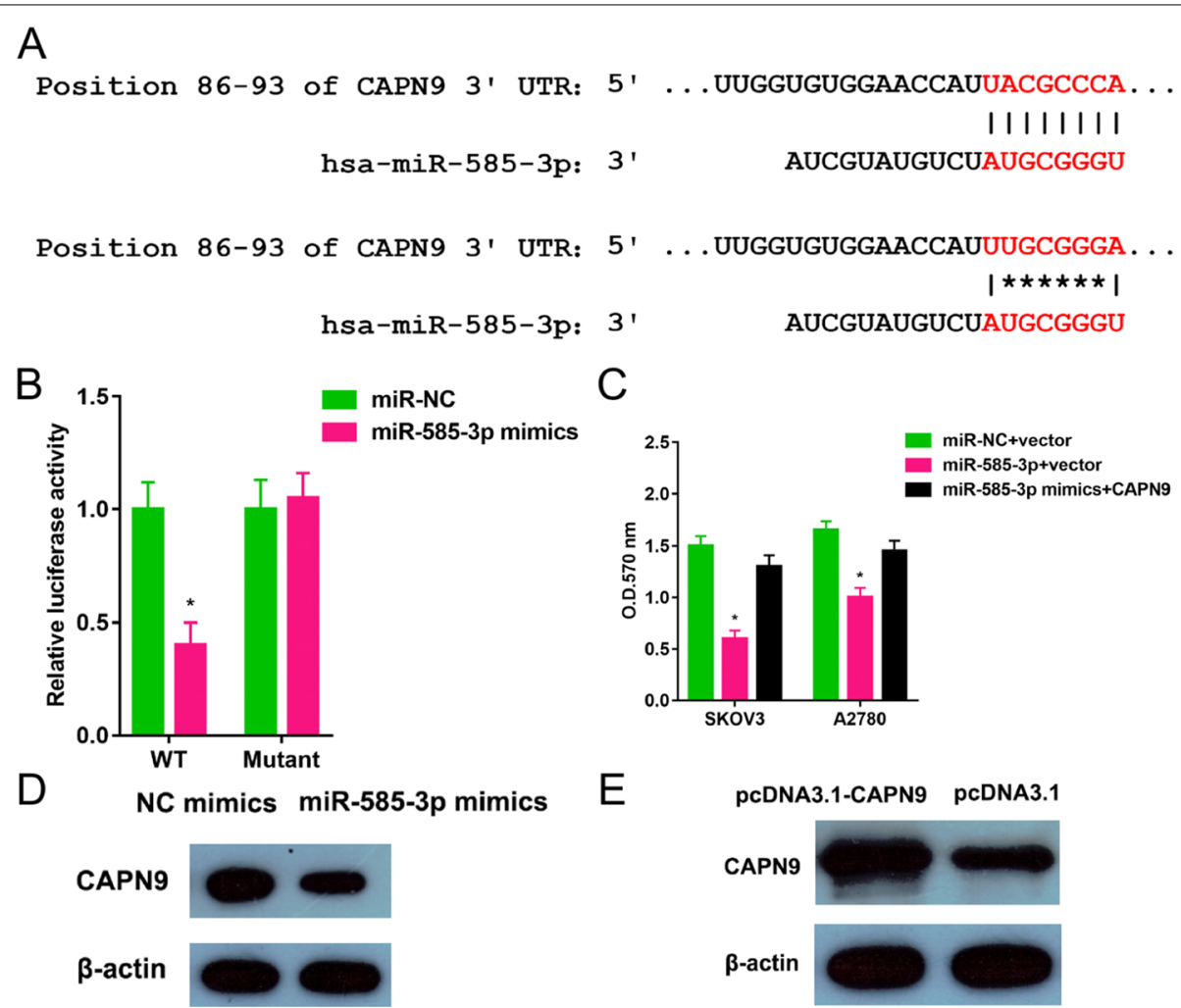

Fig. 7 miR-585-3p targeted CAPN9. A Binding site locations (positions 86-93) were listed. B miR-585-3p mimics and a plasmid containing either a wild-type or mutated 3'-UTR sequences of CAPN9 were transferred into SKOV3 cells. C MTT assay was performed. *P $<0.05$. D The amount of CAPN9 protein was determined via western blot. EThe western blot was carried out to test the CAPN9 protein levels following transfection

miR-585-3p downregulation promoted cell growth and migration of OC cell lines. These suggested that miR585-3p played an inhibitory role in HGSOC.

In this study, bioinformatics analysis indicated that the CAPN9 gene is one of the miR-585-3p targets. The results of molecular biology experiments showed that
CAPN9 was a novel downstream target of miR-585 and CAPN expression was regulated by miR-585 through direct binding to the 3'-UTR.

Calpains, a family of calcium-dependent cysteine proteases, consist of more than ten genes and contain two classes: classical calpains and non-classical calpains [17]. 
Calpains were associated with cellular processes, such as apoptosis and migration [18, 19]. Calpain-9 (encoded by CAPN9) (also known as nCL-4) is a more recently characterized member of the colpain family that was originally thought to be expressed in a digestive tract tissue-dependent manner. Gene expression of CAPN9 has turned out to be lowered in gastric cancer [20]. CANP9 is reflected in invasive breast cancer and is not expressed solely in a digestive tract specific manner [21]. In this study, CAPN9 was expressed at significantly higher levels in tumor tissues and cell lines than in normal ovarian tissue cell lines. The consequence was consisted with the result (Tumor: 1.58, Normal: 0.13) from GEPIA database (http://gepia.cancer-pku.cn/detail.php?GEPIA=GEPIA). We speculated that this may be due to different mechanisms of action mediated by the CAPN9 gene in different tumors and in different stages of development of the same tumor.

Circular RNA hsa_circRNA_102958 regulates miR$585 / \mathrm{CDC} 25 \mathrm{~B}$ to promote tumorigenesis in colorectal cancer [22] (17). Lnc01436 inhibits miR-585-3p expression, upregulates MAPK1 expression, and promotes gastric cancer progression [23]. Lnc01436, an oncogenic lncRNA in gastric cancer, regulated miR585 and FBOX11 and promoted proliferation and metastasis of GC cells [24]. LncRNA H19 regulates miR-585-3p/PIK3R3 to attenuate MPTP-induced apoptosis in Parkinson's disease [25]. The GAS6-AS1/ miR-585/EIF5A2 pathway plays an important role in HCC progression and is a potential target for therapeutic approaches in HCC [26] (21). The relationship between miR-585/CANP9 and lncRNAs has yet to be investigated. miR-585-3p acted directly on the target gene CAPN9 to promote the proliferation and migration of HGSOC.

This study was unable to investigate the relationship between miR-585-3p expression and the clinical characteristics of HGSOC patients due to the limited number of HGSOC tumor tissue samples. The relationship between miR-585-3p expression and the prognosis of HGSOC patients needs to be further studied.

\section{Conclusion}

MiR-585-3p was expressed in lower levels in ovarian cancer tissues and cell lines than in normal ovarian tissues and cell lines. Down-regulated miR-585 inhibited the growth and migration of HGSOC. miR-585-3p acted directly on the target gene CAPN9 to promote the proliferation and migration of HGSOC. Targeted treatment based on miR-585/CANP9 is beneficial to improve the therapeutic outcome of HGSOC patients.

\section{Supplementary Information}

The online version contains supplementary material available at https://doi. org/10.1186/s13048-021-00841-w.

Additional file 1: Table S1. The clinical characteristics of the patients in this study.

Additional file 2: Table $\mathbf{S 2}$. The sequence of primers in the present study.

\section{Acknowledgements}

The datasets generated and analyzed in this study are available from TargetScan and miRDB that provide free online tools and resources.

\section{Authors' contributions}

$\mathrm{XL}$ and $\mathrm{BC}$ designed the research. $\mathrm{XL}, \mathrm{GL}$, and $\mathrm{BC}$ did the experiments. $\mathrm{XL}, \mathrm{GL}$, $S L, H W$, and $B C$ did the analysis. $X L, G L, S L, H W$, and $B C$ wrote the manuscript. $X L$ and $B C$ reviewed the manuscript. All authors read and approved the final manuscript.

\section{Funding}

This work was supported by Jiangsu Provincial Key Discipline of Maternal and Child Health (2017103033)

\section{Availability of data and materials}

All data generated or analyzed during this study are included in this published article.

\section{Declarations}

Ethics approval and consent to participate

This study was approved by the ethics committee of Xuzhou Medical University Affiliated Hospital. All patients signed a written informed consent form.

\section{Consent for publication}

Not applicable.

\section{Competing interests}

The authors declare that they have no competing interests.

\section{Author details}

${ }^{1}$ Department of Gynecology, The Affiliated Hospital of Xuzhou Medical University, Jiangsu 221000, China. ${ }^{2}$ Department of Gynecology, Maternal and Child Health Care Hospital Affiliated to Xuzhou Medical University, Jiangsu 221000 China. ${ }^{3}$ Graduate School, Xuzhou Medical University, Jiangsu 221000, China.

${ }^{4}$ Xuzhou Medical University, Jiangsu 221000, China.

Received: 14 June 2021 Accepted: 29 June 2021

Published online: 08 July 2021

\section{References}

1. Ferlay J, Soerjomataram I, Dikshit R, Eser S, Mathers C, Rebelo M, Parkin DM, Forman D, Bray F. Cancer incidence and mortality worldwide: sources, methods and major patterns in GLOBOCAN 2012. Int J Cancer. 2015;136:E359-386.

2. Chen W, Zheng R, Baade PD, Zhang S, Zeng H, Bray F, Jemal A, Yu XQ, He J. Cancer statistics in China, 2015. CA Cancer J Clin. 2016;66:115-32.

3. Jayson GC, Kohn EC, Kitchener HC, Ledermann JA. Ovarian cancer. Lancet. 2014:384:1376-88.

4. Berns EM, Bowtell DD. The changing view of high-grade serous ovarian cancer. Cancer Res. 2012;72:2701-4.

5. Yuan J, Li T, Yi K and Hou M. The suppressive role of miR-362-3p in epithelial ovarian cancer. Heliyon. 2020; 6: e04258.

6. Ambros V. MicroRNA pathways in flies and worms: growth, death, fat, stress, and timing. Cell. 2003;113:673-6. 
7. Bartel DP. MicroRNAs: target recognition and regulatory functions. Cell. 2009;136:215-33.

8. Liu C, Yang J, Wu H, Li J. Downregulated miR-585-3p promotes cell growth and proliferation in colon cancer by upregulating PSME3. Onco Targets Ther. 2019;12:6525-34.

9. Chen W, Hong L, Hou C, Wang Y, Wang F, Zhang J. MicroRNA-585 inhibits human glioma cell proliferation by directly targeting MDM2. Cancer Cell Int. 2020;20:469.

10. Hu L, Wu H, Wan X, Liu L, He Y, Zhu L, Liu S, Yao H, Zhu Z. MicroRNA-585 suppresses tumor proliferation and migration in gastric cancer by directly targeting MAPK1. Biochem Biophys Res Commun. 2018;499:52-8.

11. Gerlier D, Thomasset N. Use of MTT colorimetric assay to measure cell activation. J Immunol Methods. 1986;94:57-63.

12. Rüster $B$, Grace B, Seitz $O$, Seifried E, Henschler R. Induction and detection of human mesenchymal stem cell migration in the 48-well reusable transwell assay. Stem Cells Dev. 2005;14:231-5.

13. Agarwal V, Bell GW, Nam J-W and Bartel DP. Predicting effective microRNA target sites in mammalian mRNAs. eLife. 2015; 4: e05005.

14. Chiang HR, Schoenfeld LW, Ruby JG, Auyeung VC, Spies N, Baek D, Johnston WK, Russ C, Luo S, Babiarz JE, Blelloch R, Schroth GP, Nusbaum C, Bartel DP. Mammalian microRNAs: experimental evaluation of novel and previously annotated genes. Genes Dev. 2010;24:992-1009.

15. Chen $Y$, Wang $X$. miRDB: an online database for prediction of functional microRNA targets. Nucleic Acids Res. 2020;48:D127-d131.

16. Liu W, Wang X. Prediction of functional microRNA targets by integrative modeling of microRNA binding and target expression data. Genome Biol. 2019;20:18.

17. Sorimachi H, Hata S, Ono Y. Calpain chronicle-an enzyme family under multidisciplinary characterization. Proc Japan Acad Ser B Phys Biol Sci. 2011;87:287-327.

18. Valero JG, Cornut-Thibaut A, Jugé R, Debaud A-L, Giménez D, Gillet G, Bonnefoy-Bérard N, Salgado J, Salles G, Aouacheria A, Kucharczak J. $\mu$-Calpain conversion of antiapoptotic Bfl-1 (BCL2A1) into a prodeath factor reveals two distinct alpha-helices inducing mitochondria-mediated apoptosis. PLoS One. 2012;7:e38620-e38620.

19. Cortesio CL, Boateng LR, Piazza TM, Bennin DA, Huttenlocher A. Calpainmediated proteolysis of paxillin negatively regulates focal adhesion dynamics and cell migration. J Biol Chem. 2011;286:9998-10006.

20. Peng P, Wu W, Zhao J, Song S, Wang X, Jia D, Shao M, Zhang M, Li L, Wang L, Duan F, Zhao R, Yang C, Wu H, Zhang J, Shen Z, Ruan Y, Gu J. Decreased expression of Calpain-9 predicts unfavorable prognosis in patients with gastric cancer. Sci Rep. 2016;6:29604-29604.

21. Davis J, Martin SG, Patel PM, Green AR, Rakha EA, Ellis IO, Storr SJ. Low calpain-9 is associated with adverse disease-specific survival following endocrine therapy in breast cancer. BMC Cancer. 2014;14:995-995.

22. Li R, Wu B, Xia J, Ye L, Yang X. Circular RNA hsa_circRNA_102958 promotes tumorigenesis of colorectal cancer via miR-585/CDC25B axis. Cancer Manag Res. 2019;11:6887-93.

23. Xu Y, Dong M, Wang J, Zhao W, Jiao M. LINC01436 Inhibited miR-585-3p Expression and Upregulated MAPK1 Expression to Promote Gastric Cancer Progression. Dig Dis Sci. 2021;66:1885-94.

24. Zhang Y, Yang G, He X, Chen S, Zhang F, Fang X. LINC01436, regulating miR-585 and FBXO11, is an oncogenic IncRNA in the progression of gastric cancer. Cell Biol Int. 2020;44:882-93.

25. Zhang Y, Xia Q, Lin J. LncRNA H19 Attenuates Apoptosis in MPTP-Induced Parkinson's Disease Through Regulating miR-585-3p/PIK3R3. Neurochem Res. 2020;45:1700-10.

26. Ai J, Sun J, Zhou G, Zhu T, Jing L. Long non-coding RNA GAS6-AS1 acts as a ceRNA for microRNA-585, thereby increasing EIF5A2 expression and facilitating hepatocellular carcinoma oncogenicity. Cell Cycle (Georgetown, Tex). 2020;19:742-57.

\section{Publisher's Note}

Springer Nature remains neutral with regard to jurisdictional claims in published maps and institutional affiliations.
Ready to submit your research? Choose BMC and benefit from:

- fast, convenient online submission

- thorough peer review by experienced researchers in your field

- rapid publication on acceptance

- support for research data, including large and complex data types

- gold Open Access which fosters wider collaboration and increased citations

- maximum visibility for your research: over $100 \mathrm{M}$ website views per year

At BMC, research is always in progress.

Learn more biomedcentral.com/submissions 